Review Article

\title{
Nanovaccines for cancer immunotherapy
}

\author{
Volume 4 Issue 4 - 2017 \\ Cuhananthan W Sathiyajith \\ Intelli Particle Pty Ltd,Australia
}

\section{Introduction}

Nanotechnology has expanded its horizon in to the field of medicine and has been commonly referred to by the portmanteau "Nanomedicine". Nanomedicine contributes to enhancement of human health via molecular knowledge and molecular tools. ${ }^{1}$ The field has influenced wide span of clinical applications, particularly in Cancer management. This includes drug-release and post treatment response monitoring, systems targeting cancer prevention and also patient compliance, such as anti-cancer controlled drug delivery systems. For the purpose of clarity, this article will focus on the influence of nanomedicine in the development of drug delivery systems, specifically on Nanovaccines which carry and sitespecifically deliver the antigens and monoclonal antibodies $(\mathrm{mAb})$.

\section{Cancer immunotherapy and cancer vaccines}

There are three major types of therapeutic approaches currently available to combat cancer, namely chemotherapy (which utilises small molecule agents to destroy cancer cells), radiotherapy (radio labelled small molecules) and immunotherapy. The immunotherapy is the oldest among them, which dates back to the discovery of a surgeon known as William B. Coley in 1891, who has treated his patients with bacterial products, which is now believed to have induced non-specific inflammatory responses. ${ }^{2}$ However, immunotherapy as a cancer treatment regiment has been a subject of debate for a long time due to continuous failures in clinical trials until 2013, the year in which 'Nature' has declared as 'breakthrough of the year'. Resurgence of cancer immunotherapy or Immno-Oncology (as sometimes referred to) is now evident in the advent of dendritic cell-based vaccines and immune checkpoint inhibitors. They have ushered a new line of cancer therapy and raised the hope for harnessing patients' own immune system to eradicate tumours. Cancer immunity by the human immune system is a highly complex cascade of immune response events. However, it can be attempted to be summarized in to four fold steps. Firstly, the release of antigens from tumours, secondly, presentation of tumour antigens by antigen-presenting cells (APCs), thirdly, priming and activation of T cells by activated APCs, fourthly the migration and infiltration of effectors T cells (Teff) back to the tumour and finally the recognition and destruction of tumour cells by Teff. ${ }^{3}$ In theory, each of these steps can be targeted in terms of various therapeutic approaches and can be divided into two major types, namely the passive and active therapeutic approaches. The passive immunotherapy is associated with immune checkpoint inhibitors ${ }^{4}$ which are monoclonal antibodies directed to block binding intracellular signalling surface cell receptors of $\mathrm{T}$ cells to tumour cells, thereby enabling indirect manipulation of $\mathrm{T}$ cells to combat cancer. In contrast, active approach involved direct manipulation of $\mathrm{T}$ cells in vitro. While adoptive cell transfer remains the oldest, wherein $\mathrm{T}$ cells are isolated from the body and cultured and genetically modified for specificity towards tumour associated antigens (TAAs) and subsequently reintroduced to the patient. A similar approach is undertaken with cancer vaccines, wherein TAAs are indentified and are co-administered to stimulate immune response, sometimes in the context of professional antigen presenting cells (APCs) such as dendritic cells (DCs) $)^{5}$ or viral vectors as in the case of viral vaccines. ${ }^{6}$
Correspondence: Cuhananthan W Sathiyajith, Intelli Particle Pty Ltd, I6, Carrington Road, Marrickville NSW 2204, Australia, Emailsjithcw@gmail.com

Received: May 30, 2017 | Published: July 31, 2017

\section{Nanovaccines}

Traditional approach to enhance vaccine efficacy involves using small molecule adjuvant (eg: - Alum) co-administered with antigens. However these vaccine delivery systems are limited by their ability to effectively absorb antigens and poor release characteristics and exhibited poor immune stimulatory properties. Additionally, cost per dose, lack of storage stability, also have been added to their poor efficiency. To this end, biodegradable and biocompatible synthetic and natural polymers and inorganic nanoparticles have emerged as superior alternatives. This is mainly due to their distinct characteristics such as ability to controlled release antigens with external or internal stimuli (due to acidic tumour microenvironment), established record of long term safety, controlled polymer degradation, and freedom of size assortment and encapsulation efficiency. Nanotechnology in general refers to the production and assembly of components within the range of $1-100 \mathrm{~nm}$. Harnessing the strengths of human immune system through amalgamation of cancer immunotherapy with nanotechnology has resulted in the production of Nanovaccines. These are vaccines made in nanoscale out of nanoparticles and has been rapidly emerging as a new class of vaccines that could site-specifically target the tumours, sparing normal cells. These nanoconstructs as efficient and safe vaccine delivery systems has spurred from the concept that several components imperative for vaccine efficacy could be rationally designed and assembled, optimized individually and can be incorporated in a single vehicle to induce potent immune response. Furthermore, nanoparticles as the major functional elements of these nanoconstructs could act additively or synergistically with other functional elements to provide a cooperative effect towards inducing potent immune response. Nanoparticles have been extensively investigated and widely reported over the past three decades as delivery vehicles or as nanocarriers (as they currently referred to) of traditional chemotherapeutics targeted to solid tumours. ${ }^{7-9} \mathrm{~A}$ wide span of nanoparticles, such as metallic nanoparticles, antigens conjugated to or associated with metallic nanoparticles, polymer based micelle, dendrimers, liposomes, DNA vaccines, carbon nanotubes, buckminsterfullerene, etc., have been 
investigated. Metallic nanoparticles (MeNPs), particularly iron oxides and gold are known for their non-biodegradability, structural rigidity and ease of synthesis. Moreover, many of these MeNPs have been studied for their immnunostimulatory responses such as cellular recruitment, activation of APCs, and consequent cytokine release. Furthermore, MeNPs have also been investigated in combination with antigens or as standalone nanocarriers for platinum based chemotherapeutics. ${ }^{10}$

Apart from inorganic metallic nanoparticles, polymeric nanoparticles also have been widely investigated, specifically the role of polymeric micelles, dendrimers and liposomes also have been extensively reviewed. The control of architecture of Polymeric micelles by tailoring size and shape of these particles and the subsequent utilization of these nanoconstructs to effectively deliver platinum based chemotherapeutic drugs have been widely researched. Stenzel and co-workers ${ }^{11}$ have clearly established that drugs delivered in such polymeric nanoparticles are more stable, enhance the circulation time in vivo and can by-pass drug resistance. Dendrimers also for their share have been constructed from synthetic block copolymers $^{12}$ and have been investigated extensively as vaccine delivery systems. The pioneering work of Gregoriadis and Allison ${ }^{13}$ lead to the discovery of the ability of liposomes to kindle immune responses to associated antigens paved way for new avenues in vaccine delivery systems. Similar to polymeric micelles, the design of liposomes also have freedom in selection to incorporate desired composition and preparation leading to desired architectures. Another type of novel nanomaterial that could be employed as vaccine delivery system is the nano carbon based materials, such as carbon nanotubes and Buckminster fullerenes..$^{14} \mathrm{~A}$ feature attributed to their stability in vivo, lack of inherent toxicity or immunogenicity and can be functionalised to append several antigens. ${ }^{15}$ Remodelling these nanomaterials to target the human immune system could tap the potential of patient's own immune system more effectively and could offer new opportunities to tune immunity and also to elicit strong anti-tumoral (humoral and cellular) immune responses. ${ }^{16}$ Indeed, multi-functional nanomaterials have exhibited remarkable advantages in comparison to conventional therapeutics for cancer immunotherapy.

These nanocarriers could effectively enhance the efficacy of subunit vaccines such as peptides and proteins via facilitation of presentation of APCs and T-cell Activation and realised by exploiting efficient draining of nanocarriers to lymphoid tissues in the nanoindentation holes of tumours and their prolonged tissue residence as well as controlled release of antigens and adjuvant Vaccines. However, in the recent past, efforts have also been directed to present the small molecule agonists in a polymeric platform. One such example is 'Polymap', a proprietary polymeric adjuvant platform developed by 'PsiOxus Therapeutics'. Currently, the versatile drug delivery platform has been explored in combination with nanoscaffolds delivery technology developed by 'Avidea'.

\section{Summary and outlook}

It can be stated that Nanovaccines is rapidly emerging as the poster child of cancer immunotherapy. Currently, it may be recalled that these vaccines in nanoscale designed to combat cancer is largely depend on nano particulate systems, specifically on nanocarriers. However, nanocarriers in general have inherent shortcomings and they are threefold. Firstly, the difficulty in ensuring accuracy and reproducibility of drug loading, secondly, uncontrolled release and finally possess irreversible binding characteristics. However, they are high molecular weight compounds, meaning enhanced blood circulation time which facilitates enhanced permeability and retention effect (EPR), in comparison to small molecule based nanocapsules. On the other hand, nanocapsule possesses enhanced encapsulation efficiency, a porous structure, and smart release characteristics and has been proven safe. However, they are low molecular weight. Therefore, it is plausible to envisage system acting as molecular chaperone can be constructed by the functionalization of nanocarriers with nanocapsules via covalent linker such as poly ethylene glycol. While the nanocarriers could be selected from a polymeric micelle system or inorganic material, nanocapsule could be represented by pillar (n) arenes whose biological supremacy over other macro cycles has been delineated. ${ }^{17}$ Furthermore, water soluble form of pillar ${ }^{6}$ arenes, namely carboxylated pillar ${ }^{6}$ arenes could be functionalized by antigen specific sequence or antibody drug conjugate. Additionally, nanocarriers could be encapsulated with an MRI module, to enable simultaneous tracking and treatment response monitoring. Moreover, it is also possible to functionalize one of the protruding arms with fluorophore, enabling optical imaging thus culminating in dual modal imaging. Undoubtedly, nanovaccine is rapidly emerging as the poster child of cancer immune therapy (Figure 1).

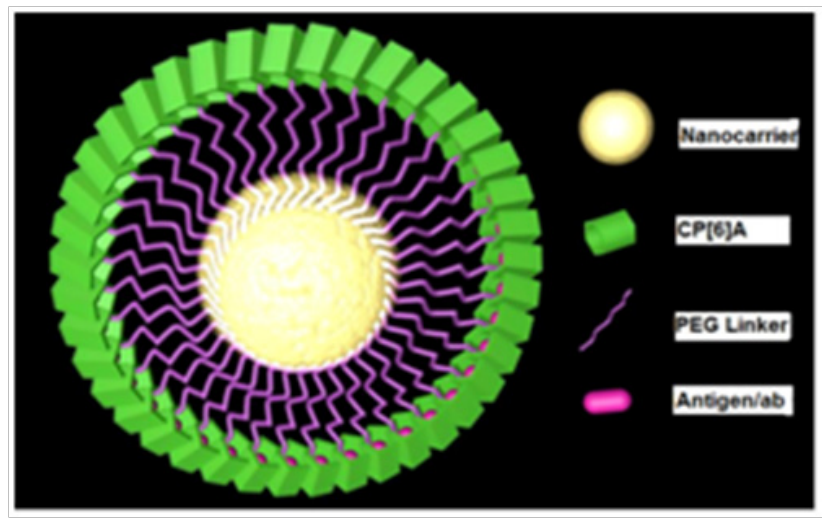

Figure I Graphical representation of molecular chaperone bearing nanocapsule encapsulated with or functionalized with vaccine antigens or monoclonal antibodies as Checkpoint inhibitors.

\section{Acknowledgments}

None.

\section{Conflicts of interest}

Author declares there are no conflicts of interest.

\section{Funding}

None.

\section{References}

1. Kostarelos K. The emergence of nanomedicine: a field in the making. Nanomedicine (Lond). 2006;1(1):1-3.

2. Coley WB. II. Contribution to the Knowledge of Sarcoma. Ann Surg. 1891;14(3):199-220.

3. Fan Y, Moon JJ. Nanoparticle Drug Delivery Systems Designed to Improve Cancer Vaccines and Immunotherapy. Vaccines (Basel). 2015;3(3):662-685.

4. Pennock GK, Chow LQM. The Evolving Role of Immune Checkpoint Inhibitors in Cancer Treatment. Oncologist. 2015;20(7):812-822.

5. Caminschi I, Maraskovsky E, Heath WR. Targeting Dendritic Cells in vivo for Cancer Therapy. Front Immunol. 2012;3:13. 
6. Saha A, Kaul R, Murakami M, Robertson ES. Tumor viruses and cancer biology: Modulating signaling pathways for therapeutic intervention. Cancer Biol Ther. 2010;10(10):961-978.

7. Aikins ME, Bazzill J, Moon JJ. Vaccine nanoparticles for protection against HIV infection. Nanomedicine(Lond). 2017;12(6):673-682.

8. Sahdev P, Ochyl LJ, Moon JJ. Biomaterials for Nanoparticle Vaccine Delivery Systems. Pharm Res. 2014;31(10):2563-2582.

9. Rosenthal JA, Chen L, Baker JL, et al. Pathogen-like particles: biomimetic vaccine carriers engineered at the nanoscale. Curr Opin Biotechnol . 2013;28:51-58.

10. Neto M, Moreira L, Kipnis A, et al. Role of Metallic Nanoparticles in Vaccinology: Implications for Infectious Disease Vaccine Development. Front Immunol . 2007;15: 8.

11. Huynh VT, Scarano W, Stenzel MH. Drug Delivery Systems for Platinum Drugs. In: (Eds.), Nanopharmaceutics. World Scientific, Singapore. 2011. p.201-241.
12. Saroja C, Lakshmi P, Bhaskaran S. Recent trends in vaccine delivery systems: A review. Int J Pharm Investig . 2011;1(2):64-74.

13. Allison AG, Gregoriadis G. Liposomes as immunological adjuvants. Nature. 1974;252(5480):252.

14. Scheinberg DA, McDevitt MR, Dao T, et al. Carbon nanotubes as vaccine scaffolds. Adv Drug Deliv Rev. 2013;65(15):2016-2022.

15. In het Panhuis M. Vaccine Delivery by Carbon Nanotubes. Chem Biol. 2003;10(10):897-898.

16. Zhao L, Seth A, Wibowo N, et al. Nanoparticle vaccines. Vaccine. 2014;32(3):327-337.

17. Sathiyajith C, Shaikh RR, Han Q, et al. Biological and related applications of pillar[n]arenes. Chem Commun . 2017;53(4):677-696. 